\title{
Spatial Correlations in Exclusion Models corresponding to the Zero Range Process
}

\author{
Urna Basu and P. K. Mohanty \\ Theoretical Condensed Matter Physics Division, Saha Institute of Nuclear Physics, \\ 1/AF Bidhan Nagar, Kolkata, 700064 India. \\ E-mail: urna.basu@saha.ac.in
}

\begin{abstract}
We show that the steady state weights of all one dimensional exclusion models which are mapped to the Zero Range Process (ZRP) can be written in a matrix product form, where the required matrices depend only on the steady state weights of ZRP. One infinite dimensional representation of these matrices which works for generic systems has also been provided. This is in contrast to the usual matrix product ansatz which does not always guarantee a solution for the dynamics dependent algebra that the matrices need to satisfy. The formulation helps us study the spatial correlations of these exclusion processes which are unreasonably difficult to obtain directly from their ZRP correspondence. To illustrate this method we reproduce certain known results, and then investigate unexplored correlations in some other model systems.
\end{abstract}

Keywords:Zero-range processes, Correlation functions (Theory), Exact results 
One dimensional driven diffusive systems with stochastic dynamics, motivated on physical grounds, are studied to understand macroscopic properties of nonequilibrium steady states [1]. These systems set a ground for the study of exotic correlations present in generic non-equilibrium systems [2]. Due to lack of generic methods analytical studies are limited to only those few systems $[3,4,5,6,6,7,8]$ for which certain specific techniques can be applied. The zero range process (ZRP) is one such exactly solvable model which displays rich non-equilibrium behaviour, despite of having a simple factorized steady state. Several one dimensional systems which are used to model dynamics of avalanches, granular systems, interface growth, polymer dynamics, various transport processes, and glasses [9] can be mapped to ZRP.

In ZRP, particles from one box hop to a neighbouring box with a rate that depends on the number of particles at the departure box. The boxes are either vacant or can accommodate arbitrary number of indistinguishable particles ignoring hard-core interaction. Several lattice models [5, 8, 10, 11] with hard-core interaction (i.e. exclusion processes) can be mapped to ZRP when vacant and occupied sites are identified as boxes and particles respectively. These exclusion processes have the same steady state weights as that of the corresponding ZRPs. However it is practically impossible to calculate the spatial correlations on the lattice as they are extremely complicated functions of ZRP variables.

In this Letter, we introduce an exclusion process on a one dimensional ring with the dynamics that is equivalent to a generic ZRP. To calculate the spatial correlations we provide a formulation where steady state of the lattice system can be expressed in a matrix product form; the particles and holes are represented by two non-commuting matrices. A specific representation of these matrices, which depends only on the steady state weight of the corresponding ZRP, is provided. The formulation can be generically used to study spatial correlations of exclusion processes having a ZRP correspondence, which is illustrated with a few examples.

The exclusion process is defined on a one dimensional periodic lattice where the sites labeled by $i=1 \ldots L$ can accommodate at best one hard-core particle. Any arbitrary configuration of the system is denoted by $\left\{s_{i}\right\}$ where $s_{i}=0,1$ corresponds to vacant and occupied sites respectively. Particles hop to a neighbouring vacant site, say to its right, according to the following dynamics,

$$
0[n] 0 \rightarrow 0[n-1] 01 \text { with rate } u(n),
$$

where a block of size $n$, i.e. an uninterrupted sequence of $n 1$ s, is denoted by $[n]$. Here, the total number of particles $N$ and thus particle density $\rho=\frac{N}{L}$ remains conserved. This dynamics does not necessarily indicate the presence of a long range interaction in the system. In fact several one dimensional driven systems [5, 11], where particles having extra degrees of freedom interact through a short range dynamics, can be described effectively by Eq. (11).

This exclusion process is equivalent to a ZRP with $N$ particles distributed in $M=L-N$ boxes with hop rate $u(n)$, provided 0 s and 1 s are referred to as boxes 
and particles respectively.

The steady state weight $P_{Z R P}\left(n_{1}, n_{2} \ldots n_{M}\right)$ of a configuration $\left\{n_{i}\right\}$ in ZRP has a product measure for any generic rate $u(n)$

$$
P_{Z R P}\left(n_{1}, n_{2} \ldots n_{M}\right)=\prod_{k=1}^{M} f\left(n_{k}\right)
$$

with $f(n)=\prod_{l=1}^{n} u(l)^{-1}$ being the weight of a box having $n$ particles [3]. Equation (2) can be used to calculate the weight of the corresponding configuration of the exclusion process as follows. Since the system is both periodic and translationally invariant, the weight $P\left(\left\{s_{i}\right\}\right)$ of a configuration $\left\{s_{i}\right\}$ can always be written as $P\left(0\left[n_{1}\right] 0\left[n_{2}\right] \ldots 0\left[n_{M}\right]\right)$ where $\left[n_{k}\right]$ is the block after $k^{t h} 0$ on the lattice. From this correspondence it is evident that

$$
P\left(\left\{s_{i}\right\}\right) \equiv P\left(0\left[n_{1}\right] \ldots 0\left[n_{M}\right]\right)=P_{Z R P}\left(n_{1}, \ldots n_{M}\right)=\prod_{k=1}^{M} f\left(n_{k}\right) .
$$

To obtain the spatial correlation functions of the exclusion process from the mapping to ZRP, one needs to express the site variables $s_{i}$ in terms of the occupation numbers $\left\{n_{k}\right\}$.

$$
s_{i}=1-\sum_{k=1}^{M} \delta_{i, g_{k}} \quad ; \quad g_{k}=\sum_{l=1}^{k}\left(1+n_{l}\right) .
$$

Clearly, even the two point correlation functions $\left\langle s_{i} s_{i+j}\right\rangle$ for arbitrary $j$ are hardly possible to calculate. Alternatively let us rewrite the steady state weight of the exclusion process on the lattice as

$$
P\left(\left\{s_{i}\right\}\right)=\operatorname{Tr}\left[X_{s_{1}} X_{s_{2}} \ldots X_{s_{L}}\right],
$$

where non-commuting matrices $X_{s_{i}}$ keep track of the position indices. This form is similar to the steady state weights assumed in Matrix Product Ansatz (MPA) [12]. However, $X_{s_{i}}$ here satisfy a unique relation [Eq. (17) below] which depends only on the single box weight $f(n)$ of the corresponding ZRP whereas MPA-matrices need to fulfill different algebraic relations depending on the concerned dynamics.

For simplicity, we use $X_{0} \equiv E$ and $X_{1} \equiv D$. From Eqs. (3) and (5) we have

$$
P\left(\left\{s_{i}\right\}\right)=\operatorname{Tr}\left[E D^{n_{1}} E D^{n_{2}} \ldots E D^{n_{M}}\right],
$$

which can be simplified further by taking $E=|\alpha\rangle\langle\beta|$, where $|\alpha\rangle$ and $\langle\beta|$ are yet to be determined. This choice of $E$, along with Eqs. (3) and (6), demands that $D$ must satisfy the following relation

$$
\left\langle\beta\left|D^{n}\right| \alpha\right\rangle=f(n) .
$$

This equation does not specify $|\alpha\rangle,\langle\beta|$ and $D$ uniquely. For this formulation to work it is sufficient to have one suitable choice of $|\alpha\rangle,\langle\beta|$ and $D$ which satisfy the above equation. A finite dimensional representation of these matrices is guaranteed only for 
certain special $f(n)$, e.g. when $f(n)$ is periodic in $n$, or when $f(n)=c^{n}$. For generic $f(n)$, however, one can use the following infinite-dimensional representation

$$
D=\sum_{i=1}^{\infty}|i\rangle\langle i+1| ; \quad\langle\beta|=\langle 1|, \quad \text { and } \quad|\alpha\rangle=\sum_{i=1}^{\infty} f(i-1)|i\rangle,
$$

where $\{|i\rangle\}$ is the standard basis in infinite dimension, i.e., $|i\rangle_{k}=\delta_{i, k}$. Both $\langle\beta|$ and $D$ are independent of the systems concerned; $\langle\beta|$ is a constant vector and $D$ is a shift operator which has the following properties

$$
\operatorname{Tr}\left[D^{m}\right]=0 ; \quad \text { and } D^{m} \sum_{i=1}^{\infty} a_{i}|i\rangle=\sum_{i=1}^{\infty} a_{i+m}|i\rangle,
$$

where $\left\{a_{i}\right\}$ are arbitrary coefficients. It is only $|\alpha\rangle$ which depends on the specific process, and can be constructed directly from the set $\{f(n)\}$. Thus, the representation (8) works for any exclusion process which can be mapped to ZRP; even when $f(n)$ does not have any functional dependence on $n$.

The first task is to calculate the partition function. This is done in the grand canonical ensemble (GCE) by associating the fugacity $z$ with particles $(D)$ resulting in $Z_{L}(z)=\operatorname{Tr}\left[C^{L}\right]$ where $C=z D+E$. The weight of the configuration having no vacant site is $\operatorname{Tr}\left[D^{L}\right]=0$. Thus, $Z_{L}(z)$ is the sum of the weights of all other configurations with at least one vacant site.

$$
Z_{L}(z)=\sum_{n=1}^{L} \operatorname{Tr}\left[(z D)^{n-1} E C^{L-n}\right]=\sum_{n=1}^{L}\left\langle\beta\left|C^{L-n}(z D)^{n-1}\right| \alpha\right\rangle .
$$

The partition function can be calculated explicitly for some simple forms of $f(n)$. For arbitrary $f(n)$, however, it is better to use the following generating function

$$
\mathrm{Z}(z, \gamma)=\sum_{L=1}^{\infty} \gamma^{L} Z_{L}(z)=\left\langle\beta\left|\frac{\gamma}{\mathcal{I}-\gamma C} \frac{1}{\mathcal{I}-\gamma z D}\right| \alpha\right\rangle,
$$

where $Z_{L}$ is the coefficient of $\gamma^{L}$ in the series expansion of $Z_{3}(z, \gamma)$. Instead, Z $(z, \gamma)$ can be interpreted as the partition function of the system in variable length ensemble (VLE). Together $z$ and $\gamma$, here, determine the macroscopic variables

$$
\langle N\rangle=\frac{z}{\mathrm{Z}} \frac{\partial \mathrm{Z}}{\partial z} \quad \text { and } \quad\langle L\rangle=\frac{\gamma}{\mathrm{Z}} \frac{\partial \mathrm{Z}}{\partial \gamma} .
$$

In the thermodynamic limit $\langle L\rangle \rightarrow \infty$, where VLE is expected to be equivalent to GCE, one of the variables (say $\gamma$ ) becomes a function of the other one $(z)$. Details of this equivalence will be discussed elsewhere.

Any $n$-point correlation function can be expressed easily in terms of holes denoted by $\bar{s}_{i}=1-s_{i}$. For example $\left\langle\bar{s}_{i}\right\rangle=\sum_{L} \operatorname{Tr}\left[(\gamma E)(\gamma C)^{L-1}\right]$, can be written as

$$
\left\langle\bar{s}_{i}\right\rangle=G^{(1)}(z, \gamma)=\frac{\gamma}{Z}\left\langle\beta\left|\frac{1}{\mathcal{I}-\gamma C}\right| \alpha\right\rangle .
$$

The density $\rho(z, \gamma)=1-G^{(1)}(z, \gamma)$, obtained from above, is same as that calculated using Eq. (12) in the thermodynamic limit. Since spatial indices do not appear in $\left\langle\bar{s}_{i}\right\rangle$ 
it can as well be obtained directly from the ZRP correspondence. The advantage of this matrix formulation will be clear in the calculation of higher order correlation functions which carry these indices.

The 2-point correlation functions $G_{j}^{(2)}=\left\langle\bar{s}_{i} \bar{s}_{i+j}\right\rangle$ are written as

$$
G_{j}^{(2)}(z, \gamma)=\frac{\gamma^{j+1}}{\mathrm{Z}}\left\langle\beta\left|C^{j-1}\right| \alpha\right\rangle\left\langle\beta\left|\frac{1}{\mathcal{I}-\gamma C}\right| \alpha\right\rangle=\gamma^{j}(1-\rho)\left\langle\beta\left|C^{j-1}\right| \alpha\right\rangle .
$$

All other $(n+1)$-point correlations can be expressed in terms of $G^{(1)}$ and $G_{j}^{(2)} \mathrm{s}$,

$$
G_{j_{1} \ldots j_{n}}^{(n+1)}=\left\langle\bar{s}_{i} \bar{s}_{i+j_{1}} \bar{s}_{i+j_{1}+j_{2}} \ldots \bar{s}_{i+j_{1}+\ldots j_{n}}\right\rangle=G^{(1)} \gamma^{n} \prod_{i=1}^{n}\left\langle\beta\left|(\gamma C)^{j_{i}-1}\right| \alpha\right\rangle=\frac{1}{G^{(1)^{n-1}}} \prod_{i=1}^{n} G_{j_{i}}^{(2)} .
$$

To evaluate $\mathrm{Z}(z, \gamma)$ we need to know $\langle\beta|(\mathcal{I}-\gamma C)^{-1}$ and $(I-\gamma z D)^{-1}|\alpha\rangle$. The later can be obtained from Eq. (9) and it can be shown that,

$$
\langle\beta| \frac{1}{I-\gamma C}=\sum_{k=1}^{\infty}\langle k| \frac{(\gamma z)^{k-1}}{1-\gamma F(\gamma z)} .
$$

Here, $F(w)=\sum_{n} w^{n} f(n)$ is nothing but the single-box grand partition function of the corresponding ZRP with fugacity $w$. Using Eqs. (91) and (15) in (11) we have

$$
\mathrm{Z}(z, \gamma)=\frac{\gamma\left[F(\gamma z)+\gamma z F^{\prime}(\gamma z)\right]}{1-\gamma F(\gamma z)}
$$

The advantage of the use of VLE is clear from the above simple and closed form expression of the partition function that we obtain for the lattice system. Equation (15) can also be used along with Eqs. (16) and (13) to obtain the average density in VLE,

$$
\rho(z, \gamma)=\frac{\gamma z F^{\prime}(\gamma z)}{F(\gamma z)+\gamma z F^{\prime}(\gamma z)}
$$

Higher order correlation functions can be calculated from $G_{m}^{(2)}$ with arbitrary $m$ for which one requires

$$
\left\langle\beta\left|C^{m}\right| \alpha\right\rangle=\left\{\gamma^{m}\right\}\left\langle\beta\left|\frac{1}{\mathcal{I}-\gamma C}\right| \alpha\right\rangle=\left\{\gamma^{m}\right\} \frac{F(\gamma z)}{1-\gamma F(\gamma z)} .
$$

Here, $\left\{x^{n}\right\} g(x)$ denotes the coefficient of $x^{n}$ in the series expansion of $g(x)$. To proceed further $F(w)$ needs to be specified. In the following, we illustrate this formalism with some examples.

Our first example is the restricted exclusion process (RASEP) [8] defined on a one dimensional periodic lattice with hardcore particles where a particle is allowed to move to a neighbouring vacant site, say to its right, if it is preceded by at least $\mu$ particles. It has been shown, that RASEP and several variations of it undergo a phase transition from an absorbing state to an active phase as the density is increased beyond a critical value $\rho_{c}=\frac{\mu}{1+\mu}$. Exact correlation functions for RASEP are obtained by using a generalization of MPA. Let us reproduce these results using the procedure sketched 
here, as these models can be mapped to a ZRP [7] with $u(n)=\Theta(n-\mu)$, where $\Theta(x)$ is the Heaviside theta function.

The simplest case $\mu=1$ corresponds to the dynamics $110 \rightarrow 101$, which is also a special case of some other models studied earlier [13] in different contexts. In the following we consider only this case; generalization to $\mu>1$ is straightforward. Here, the single box weight is $f(n)=\Theta(n)$ and correspondingly $F(w)=\frac{w}{1-w}$. Thus, in this case, $|\alpha\rangle=(0,1,1 \ldots)^{T}$. Since $\langle\beta \mid \alpha\rangle=0$, weight of any configuration with two or more consecutive 0s vanishes identically, as observed in the steady state of RASEP.

Using Eq. (16), the partition function of RASEP is given by

$$
\mathrm{Z}(z, \gamma)=\frac{\gamma^{2} z(2-\gamma z)}{\left(1-\gamma z-\gamma^{2} z\right)(1-\gamma z)}
$$

We have checked that $\left\{\gamma^{L}\right\} Z+z^{L}$ is the GCE partition function obtained earlier [14] using MPA. The extra factor $z^{L}$ is the weight of the configuration $\left\{s_{i}=1\right\}$ which does not have a ZRP equivalence. Clearly Z has to be supplemented by Eq. (12). For any given $z$, the average system size $\langle L\rangle$ can be varied by varying $\gamma$ and the thermodynamic limit $\langle L\rangle \rightarrow \infty$ is reached as $\gamma$ approaches $\gamma^{*}=\frac{\sqrt{z^{2}+4 z}-z}{2 z}$. In this limit $\rho(z)=\frac{1}{2-z \gamma^{*}}=\frac{1}{2}\left[1+\sqrt{\frac{z}{4+z}}\right]$, and the $(n+1)$-point correlation function

$$
\left\langle s_{i} s_{i+1} \ldots s_{i+n}\right\rangle=\sum_{L=n+1}^{\infty} \gamma^{L} \operatorname{Tr}\left[D^{n+1} C^{L-n-1}\right]=\frac{\left[z \gamma^{*}\right]^{n}}{2-z \gamma^{*}}=\rho\left[\frac{2 \rho-1}{\rho}\right]^{n} .
$$

Both the density-fugacity relation and the correlation functions, calculated in VLE, are same as those obtained earlier in GCE [8].

We close the discussion on RASEP with the comment that an alternative finite dimensional representation $D=\left(\begin{array}{ll}0 & 1 \\ 0 & 1\end{array}\right),|\alpha\rangle=\left(\begin{array}{l}0 \\ 1\end{array}\right)$ and $\langle\beta|=\left(\begin{array}{ll}1 & 0\end{array}\right)$ which satisfy Eq. (17) is identical to the matrices obtained in [8] using MPA.

As a second example we study another one dimensional system [11] where particles have extra degrees of freedom, namely spin. Each site $i$ of the periodic lattice is either vacant or occupied with one + or - kind of particle; correspondingly $\tau_{i}=0, \pm 1$. These particles interact through an Ising-like interaction $H=-\epsilon / 4 \sum_{i} \tau_{i} \tau_{i+1}$ which is reflected in the dynamics:

$$
+-\stackrel{1-\Delta H}{\longrightarrow}-+\quad ; \quad+0 \stackrel{\alpha}{\rightarrow} 0+\quad ; \quad 0-\stackrel{\alpha}{\rightarrow}-0 .
$$

This model, hereafter referred to as EKLS, is an extension of an exactly solvable system studied by Katz-Lebowitz-Spohn [6]. It has been shown [11] that EKLS can be mapped to ZRP approximately, ignoring the spin indices, i.e., 0s considered as boxes and both $+\mathrm{s}$ and $-\mathrm{s}$ are considered as particles. The new site variables are now $s_{i}=\tau_{i}^{2}$. ZRP hop rate for a system having same numbers of + and - particles is

$$
u(n)=J_{n}=\frac{v+\epsilon}{v^{3}}\left(1+\frac{b(\epsilon)}{n}\right) ; b(\epsilon)=\frac{3 v(2+\epsilon)+6 \epsilon}{4(v+\epsilon)} ; v=\sqrt{\frac{1+\epsilon}{1-\epsilon}}+1
$$

where $J_{n}$ is the current through a block of size $n$ to the leading order in $1 / n$. The ZRP mapping is known to be exact [15] for the special case $\epsilon=0$. 


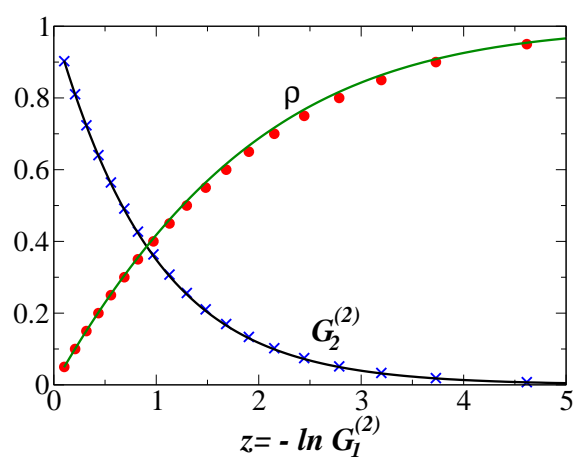

Figure 1. (Color online) $\rho$ and $G_{2}^{(2)}$ as a function of $z=-\ln G_{1}^{(2)}$. Results from numerical simulations (symbols) of EKLS for $\epsilon=-8 / 17$ with $L=1000$ and $\alpha=1$ are compared with theoretical calculations (solid line).

As $\epsilon$ is increased beyond a critical value $4 / 5$, where $b=2$, a phase separation transition occurs in EKLS model for large densities. Extensive numerical studies have provided evidence [11] of such a transition. However, to the best of our knowledge, the spatial correlations of these models have not been studied yet. Let us use the formalism discussed here to calculate these correlations and compare them with those obtained from numerical simulations. Although the spatial correlations can be found for any arbitrary $b(\epsilon)$, we choose to work with $b=1$ corresponding to $\epsilon=-8 / 17$ (antiferromagnetic interaction) where it is possible to find simple closed form expressions. Then ignoring the prefactor, which only redefines the fugacity, we have $u(n)=(n+1) / n$. Thus $f(n)=1 /(n+1)$ and correspondingly $F(w)=-\ln (1-w) / w$. The partition function takes a simple form

$$
\mathrm{Z}=\frac{\gamma z}{(1-\gamma z)(z+\ln (1-\gamma z))}
$$

In the thermodynamic limit $\langle L\rangle \rightarrow \infty$, which corresponds to $\gamma^{*}=z^{-1}\left(1-e^{-z}\right)$, the density is $\rho=1-z /\left(e^{z}-1\right)$. The two-point correlation functions (14) are then,

$$
G_{j}^{(2)}(z)=\gamma^{* j}(1-\rho) \sum_{i=1}^{j} \frac{z^{j}}{i(j-i) !} \int_{0}^{\infty} d \lambda e^{-\lambda z}(\lambda)_{j-i},
$$

where $(\lambda)_{l}=\lambda(\lambda+1) \ldots(\lambda+l-1)$ is the Pochhammer symbol. Explicitly for $j=1,2$

$$
G_{1}^{(2)}=e^{-z}, \quad G_{2}^{(2)}=\frac{\left(1-e^{-z}\right)(2+z)}{2 z e^{z}} .
$$

Usually the correlation functions in GCE are compared with those in the canonical ensemble (having fixed density $\rho=N / L$ ) by expressing $z$ in terms of $\rho$. However, here it is simpler to use $z=-\ln G_{1}^{(2)}$ and express $\rho$ and other correlation functions in terms of $G_{1}^{(2)}$. In Fig. 1 these results are compared with the 2-point correlation functions obtained from the numerical simulations of EKLS with different densities $\rho$. An excellent match indicates that the mapping of EKLS to ZRP is a good approximation. 
In summary, we have studied an exclusion process on a one dimensional ring where a particle moves to its rightward vacant neighbour with a rate that depends on the size of the block to which the particle belongs. The model has a natural correspondence to zero range process but positional ordering of the particles is lost in this mapping. Thus the spatial correlations of the exclusion process are unreasonably difficult to calculate directly from the steady state weights of ZRP. A method is introduced to obtain the same, by rewriting the steady state weights of the exclusion process in matrix product form. The required matrices are constructed explicitly using only the single box weight of the corresponding ZRP. The method is illustrated using the example of RASEP. Since, several one dimensional exclusion processes are known to have ZRP correspondence, this formulation can be used to study unexplored spatial correlations in such systems. As immediate applications, spatial correlations in EKLS model (discussed here), Tonk's gas and RASEP with parallel dynamics (to be discussed elsewhere) have been studied. We believe that this method is a useful tool for the study of correlations in one dimensional non-equilibrium steady states.

We are grateful to Deepak Dhar for his useful comments on the manuscript. U.B. would like to acknowledge thankfully the financial support of the Council of Scientific and Industrial Research, India (Grant No. SPM-07/489(0034)/2007).

\section{References}

[1] B. Schmittmann and R. K. P. Zia, Statistical Mechanics of Driven Diffusive Systems ed. C. Domb and J. L. Lebowitz, 1995 Academic Press, New York

[2] Nonequilibrium Statistical Mechanics In One Dimension, ed. V. Privman, 1997 Cambridge University Press

[3] F. Spitzer, Interaction of Markov processes, 1970 Adv. Math. 5

[4] B. Derrida et. al., Exact solution of a one-dimensional asymmetric exclusion model using a matrix formulation, 1993 J. Phys. A: Math. Gen. 26, 1493.

[5] P. F. Arndt, T. Heinzel and V. Rittenberg, Spontaneous breaking of translational invariance in onedimensional stationary states on a ring, 1998 J. Phys. A: Math. Gen. 31, L45 ; ibid, Spontaneous Breaking of Translational Invariance and Spatial Condensation in Stationary States on a Ring. I. The Neutral System, 1999 J. Stat. Phys. 97, 1

[6] S. Katz, J. L. Lebowitz and H. Spohn, Nonequilibrium steady states of stochastic lattice gas models of fast ionic conductors, 1984 J. Stat. Phys. 34, 497

[7] K. Jain, Simple sandpile model of active absorbing state transitions, 2005 Phys. Rev. E 72, 017105

[8] U. Basu and P. K. Mohanty, Active absorbing state phase transition beyond directed percolation: A class of exactly solvable models, 2009 Phys. Rev. E 79, 041143

[9] M. R. Evans and T. Hanney, Nonequilibrium statistical mechanics of the zero-range process and related models, 2005 J. Phys. A: Math. Gen. 38, R195

[10] E. Levine et. al., Traffic jams and ordering far from thermal equilibrium, 2004 Physica A 340, 636 ; G. Schnherr and G. M. Schutz, Exclusion process for particles of arbitrary extension: hydrodynamic limit and algebraic properties, 2004 J. Phys. A: Math. Gen. 37 8215; A. Kunwara et. al., Collective traffic-like movement of ants on a trail: dynamical phases and phase transitions, 2004 Jnl. Phys. Soc. Jpn. 73, 2979

[11] Y. Kafri et. al., Phase-separation transition in one-dimensional driven models, 2003 Phys. Rev. E 68, 035101(R); M. R. Evans et. al., Modelling one-dimensional driven diffusive systems by the Zero-Range Process, 2004 Euro. Phys. J. B 41, 223 
[12] R. A. Blythe and M. R. Evans, Nonequilibrium steady states of matrix-product form: a solver's guide, 2007 J. Phys. A : Math. Theor. 40, R333

[13] H. M. Koduvely and D. Dhar, A Model of Subdiffusive Interface Dynamics with a Local Conservation of Minimum Height, 1998 J. Stat. Phys. 90, 57; V. Popkov and G. M. Schütz, Steady-state selection in driven diffusive systems with open boundaries, 1999 Europhys. Lett., 48, 257

[14] Note that in [8] the fugacity was taken as $z^{2}$.

[15] Y. Kafri et. al., Criterion for Phase Separation in One-Dimensional Driven Systems, 2002 Phys. Rev. Lett. 89, 035702 